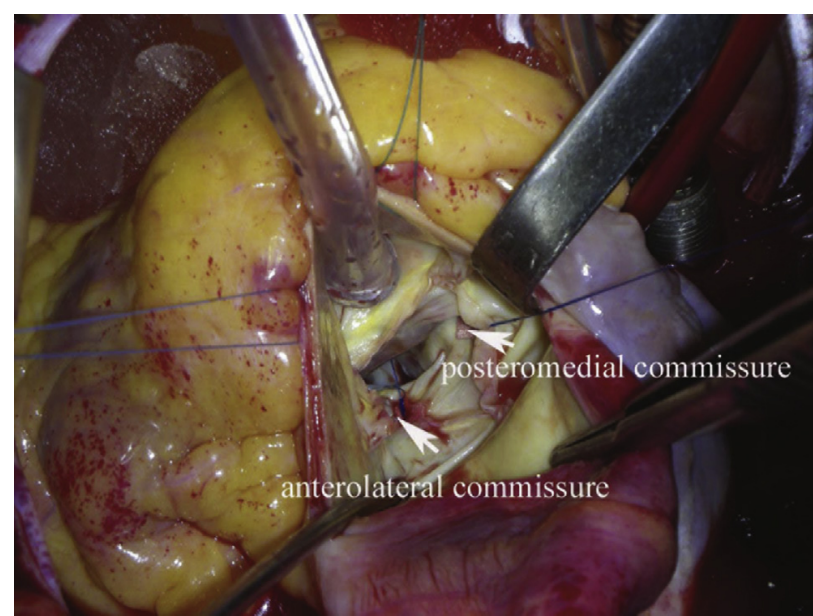

FIGURE 2. Suture annuloplasty through a transaortic approach.

regurgitation at the ninth month after the procedure. The other 2 patients had recurrence of mild regurgitation at the third and 30th months. Freedom from mitral regurgitation was $86.9 \%$.

\section{DISCUSSION}

Suture annuloplasty can maintain the physiologic motion and spatial structure of the mitral annulus without prosthetic ring. Commissural suture, a type of suture annuloplasty, can effectively recover the mitral valve function by decreasing the length of the posterior annulus. ${ }^{4}$ Our data showed good results of the commissural suture for moderate FMR. We could get excellent mitral valve exposure through the transaortic approach when the diameter of aortic annulus was enlarged. A retractor was used to withdraw the chordae to reveal the commissural area and avoid entanglement. Commissural suture performed through the transaortic approach was just as easy as through a routine approach. Nevertheless, the technique requires experience when executing it on an aortic annulus of normal or small size diameter. Transaortic repair was suitable, especially when upper median sternotomy or transverse parasternal incision over the third intercostal space was used. The advantages included no bleeding from an atrial incision and no extensive release of adhesions in reoperations. ${ }^{5}$ It is thus safe, effective, and convenient to repair moderate FMR by commissural suture through transaortic approach when the procedure of aortic valve or aortic root replacement is performed.

We thank Drs Saie Shen, Yuan Sun, and Yin Cai and Mss Huihua Chen and $\mathrm{Yu} \mathrm{Su}$ for their excellent clinical support and follow-up data collection. We thank Dr Ting Zhang for the language revision. We thank Mr Dai Yang for the diagram.

\section{References}

1. Enriquez-Sarano M, Akins CW, Vahanian A. Mitral regurgitation. Lancet. 2009; 373:1382-94

2. Wan CK, Suri RM, Li Z, Orszulak TA, Daly RC, Schaff HV, et al. Management of moderate functional mitral regurgitation at the time of aortic valve replacement: is concomitant mitral valve repair necessary? J Thorac Cardiovasc Surg. 2009;137: 635-40.

3. Suri RM, Aviernos JF, Dearani JA, Mahoney DW, Michelena HI, Schaff HV, et al Management of less-than-severe mitral regurgitation: should guidelines recommend earlier surgical intervention? Eur J Cardiothorac Surg. 2011;40:496-502.

4. Fundarò P, Tartara PM, Villa E, Fratto P, Campisi S, Vitali EO. Mitral valve repair: is there still a place for suture annuloplasty? Asian Cardiovasc Thorac Ann. 2007; $15: 351-8$

5. Koizumi J, Nakajima T, Izumoto H, Ohsawa S, Ishihara K, Kawazoe K Transaortic mitral valve repair combined with a modified Bentall procedure in a patient with Marfan's syndrome: report of a case. Surg Today. 2004;34:774-6.

\title{
An alternative technique for surgical repair of pulmonary dissection
}

\author{
Gianfranco Montesi, MD, ${ }^{\mathrm{a}}$ Sabina Caciolli, MD, ${ }^{\mathrm{b}}$ Manlio Acquafresca, MD, ${ }^{\mathrm{b}}$ and \\ Sandro Gelsomino, $\mathrm{MD}, \mathrm{PhD},{ }^{\mathrm{a}}$ Florence, Italy
}

Pulmonary artery dissection (PAD) is a rare but lifethreatening event that usually occurs with other congenital

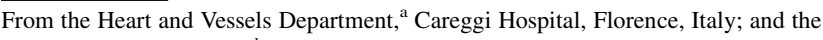
Radiology Department, ${ }^{\mathrm{b}}$ Careggi Hospital, Florence, Italy.

Disclosures: Authors have nothing to disclose with regard to commercial support.

Received for publication May 9, 2013; revisions received July 30, 2013; accepted for publication Sept 6, 2013; available ahead of print Oct 30, 2013.

Address for reprints: Sandro Gelsomino, MD, PhD, Department of Heart and Vessels,

Careggi Hospital, Largo Brambilla 3, 50134, Florence, Italy (E-mail: sandro.

gelsomino@libero.it).

J Thorac Cardiovasc Surg 2014;147:527-9

$0022-5223 / \$ 36.00$

Copyright (c) 2014 by The American Association for Thoracic Surgery

http://dx.doi.org/10.1016/j.jtcvs.2013.09.019
}

cardiac defects or as a complication of chronic pulmonary arterial hypertension. ${ }^{1}$ Other causes of PAD include connective tissue disorders, right heart endocarditis, amyloidosis, trauma, and severe atherosclerosis. Only few cases of PAD treated surgically have been reported in the literature, ${ }^{2-4}$ and no definitive approach has been established. We describe an alternative technique for surgical repair of PAD.

\section{CLINICAL SUMMARY}

A 46-year-old patient with Ehlers-Danlos syndrome who had undergone Bentall composite aortic root replacement for type A aortic dissection 10 years previously was 

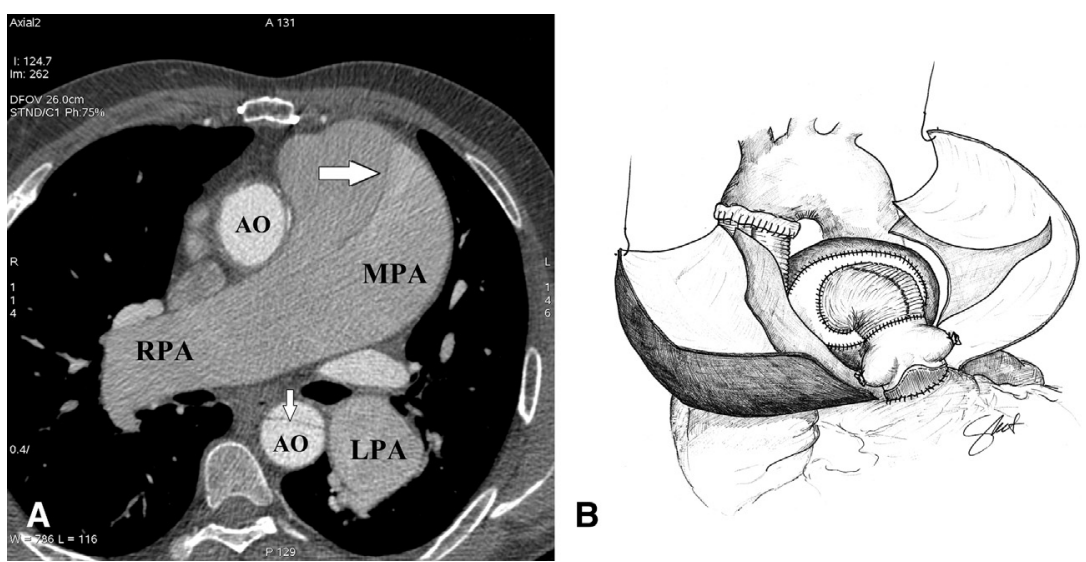

FIGURE 1. A, Preoperative computed tomographic axial view showing a dilatation of the main pulmonary artery involving both pulmonary branches, with an intimal flap in the pulmonary trunk (large arrow). An intimal flap in the descending aorta (small arrow) is also visible. B, Schematic drawing of the surgical procedure. The pulmonary root was replaced with a composite stentless xenograft, with Dacron polyester fabric graft interposition. The pulmonary artery bifurcation was reconstructed with a pericardial patch as described in the text. $A O$, Aorta; $M P A$, main pulmonary artery; $L P A$, left pulmonary artery; $R P A$, right pulmonary artery.

referred to our facility with exertional chest pain and dyspnea. Transthoracic echocardiography revealed an enlarged pulmonary artery with moderate to severe pulmonary valve incompetence and an intimal dissection flap of the main pulmonary artery (MPA). Diagnosis of PAD was confirmed by computed tomography (Figure 1, A), which also showed slight enlargement of the pulmonary branches (right pulmonary artery $3.8 \mathrm{~cm}$, left pulmonary artery 3.6 $\mathrm{cm})$. The decision was therefore made for emergency surgery.

Cardiopulmonary bypass was established through cannulation of the right axillary artery (through an 8-mm Dacron polyester fabric side graft prosthesis) and femoral vein, followed by resternotomy and careful dissection of pericardial adhesions. The patient was cooled to a rectal temperature of $30^{\circ} \mathrm{C}$. The ascending aortic graft was crossclamped, and cold crystalloid cardioplegia was delivered in an antegrade manner to achieve cardiac arrest. The left ventricle was vented with a cannula placed through the right superior pulmonary vein. The aneurysmal pulmonary artery was incised longitudinally and inspected. An intimal tear was located in the main trunk, running from $2 \mathrm{~cm}$ above the annulus to the pulmonary bifurcation and involving the origin of the left pulmonary artery. The dissected MPA was excised, and the distal line of excision was extended laterally along the anterior wall of the pulmonary bifurcation on each side, including the entire dissected area.

A vent was placed into the right and left pulmonary arteries through the resulting defect, which was repaired with a bovine pericardial patch sutured with 5-0 Prolene running suture (Ethicon, Inc, Somerville, NJ). At surgical inspection the pulmonary valve was markedly insufficient, with a thickened anterior leaflet, and it was therefore deemed irreparable. After excision of the pulmonary valve, a 29-mm Freestyle stentless porcine bioprosthesis (Medtronic, Inc, Minneapolis, Minn) was implanted as root replacement with three 3-0 Prolene running sutures.

Finally, a 28-mm Dacron polyester fabric graft (DuPont, Wilmington, Del) was interposed between the neopulmonary root and the pericardial patch with a 4-0 Prolene running suture (Figure 1, B). After cardiopulmonary bypass was discontinued, the operation was routinely completed. Intraoperative echocardiography confirmed the proper positioning and function of the pulmonary valve and trunk graft.

The postoperative pulmonary hypertension was treated with diuretics, $\beta$-blockers, and angiotensin receptor blockers. After a transient rise in pulmonary arterial pressure, this treatment led to a gradual and stable normalization.

Computed tomography (Figure 2) carried out at 19 postoperative months showed correct positioning of the graft with no sign of contrast leakage.

\section{DISCUSSION}

PAD involving the MPA is rare and always occurs in a site of pulmonary aneurysm or dilatation. It is usually diagnosed in patients with pulmonary hypertension secondary to Eisenmenger syndrome, ${ }^{3}$ but it may also be associated with connective tissue disorders without artery hypertension. ${ }^{1}$ Once diagnosed, PAD may necessitate emergency surgery to prevent lethal bleeding, because the false lumen tends to rupture rather than to develop a reentry site, as is usual in aortic dissection.

Nonetheless, there are still no clear guidelines for surgical indication, and some authors ${ }^{5}$ recommend surgery for patients with or without symptoms when hemodynamic 


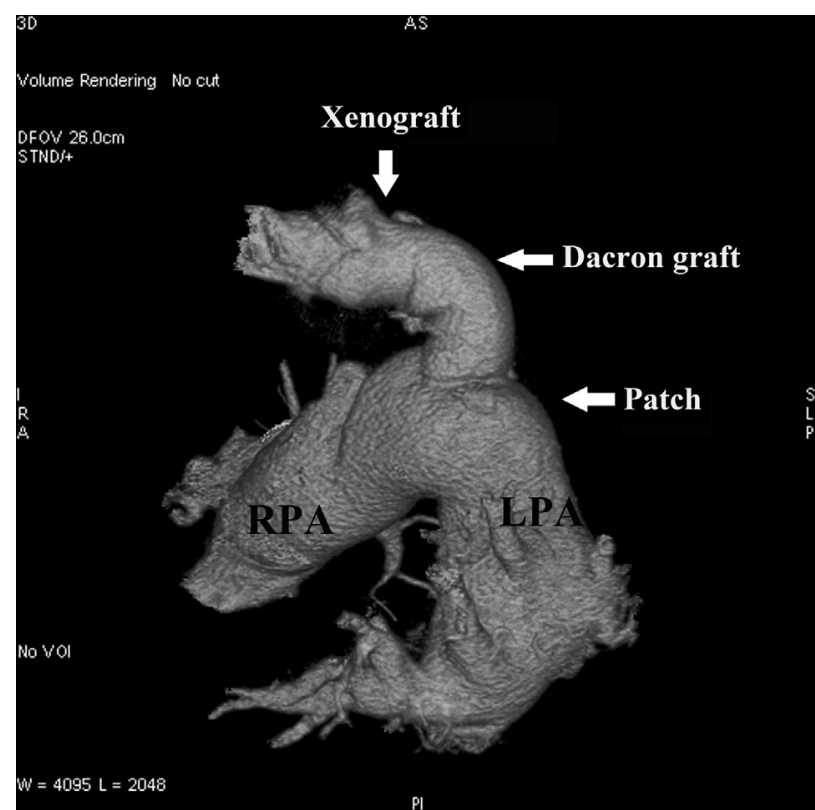

FIGURE 2. Computed tomographic 3-dimensional reconstruction showing the correct positioning of the graft and the absence of contrast agent leakage. $R P A$, Right pulmonary artery; $L P A$, left pulmonary artery.

parameters are affected by a PAD, whereas for those with stable, asymptomatic disease, they suggest conservative treatment and echocardiographic follow-up.

The literature contains only a small number of reports of patients with PAD undergoing surgery, and none with significant pulmonary valve regurgitation necessitating valve replacement. $^{2-4}$

Surgical options for pulmonary root and valve replacement include a full root homograft, a stentless xenograft root, or Dacron polyester fabric tube graft with a stented bioprosthesis sewn into it. Senbaklavaci and colleagues ${ }^{2}$ described a case of rupture and dissection in pulmonary artery aneurysm in a 34-year-old woman affected by primary pulmonary hypertension. The patient underwent successful T-shaped prosthetic graft replacement of the central pulmonary artery by means of the inclusion technique. In our patient, because of the dense adhesions between the aortic graft and the hilar pulmonary arteries, it was impossible to carry out a complete dissection of these vessels with very thin and fragile walls. We therefore could not perform a T-shaped prosthetic graft replacement as initially planned. Because of this, and also because the central pulmonary artery and its branches were only slightly enlarged, we decided simply to excise the anterior wall of the pulmonary bifurcation involved in the dissection and to close the resulting defect with a pericardial patch.

Furthermore, because in our case the aortic annulus was hugely dilated and an adequately sized homograft was not available, a stentless xenograft was chosen for its proven enhanced features. Because the diameter of the host annulus was greater than the diameter of the xenograft, special care was taken while passing sutures by taking slightly wider intervals (1-2 mm) in the host rather than the xenograft. This resulted in circumferential plication of the annulus when the sutures were tied.

Finally, the stentless xenograft was too short to replace the MPA, and the strong adhesions did not allow direct connection of the MPA to the patch. A short segment of Dacron polyester fabric tube graft was therefore interposed between the bioprosthetic root and the pericardial patch.

In conclusion, composite Freestyle stentless xenograft root replacement with Dacron polyester fabric graft interposition, in association with pulmonary artery bifurcationplasty, proved an effective and safe approach when other options were not available. Nonetheless, longer follow-up is necessary to confirm long-term performance and durability of this technique.

We gratefully acknowledge Dr Judith Wilson for the English revision of the manuscript.

\section{References}

1. Bakir I, Degrieck I, Lecomte P, Coddens J, Foubert L, Heyse A, et al. Endovascular treatment of concomitant patent ductus arteriosus and type B aortic dissection in a patient with pulmonary artery dissection. J Thorac Cardiovasc Surg. 2006;132: 438-40.

2. Senbaklavaci O, Kaneko Y, Bartunek A, Brunner C, Kurkciyan E, Wunderbaldinger $\mathrm{P}$, et al. Rupture and dissection in pulmonary artery aneurysms: incidence, cause, and treatment-review and case report. J Thorac Cardiovasc Surg. 2001;121:1006-8

3. Rousou AJ, Haddadin A, Badescu G, Geirsson A. Surgical repair of pulmonary artery dissection. Eur J Cardiothorac Surg. 2010;38:805.

4. Guo Y, Huang H, Chen W, Yang K, Wang W. A case of pulmonary artery dissection. Eur J Echocardiogr. 2011;12:E32.

5. Li Q, Wang A, Li D, Zou C, Zhang H, Wang Z, et al. Images in cardiovascular medicine. Dissecting aneurysm of the main pulmonary artery: a rare complication of pulmonary balloon valvuloplasty diagnosed 1 month after the procedure. Circulation. 2009;119:761-3. 